\title{
NIVELES DE INSATISFACCIÓN DEL USUARIO EXTERNO EN EL INSTITUTO NACIONAL DE SALUD DEL NIÑO DE LIMA, PERÚ
}

\author{
Roberto Shimabuku1,2,a, Luis Huicho ${ }^{1,2,3, a}$, Danitza Fernández ${ }^{1,2, b}$, Graciela Nakachi ${ }^{1,2, b}$, \\ Ruth Maldonado ${ }^{1, c}$, Armando Barrientos ${ }^{1, d}$
}

RESUMEN

Objetivos. Determinar los cambios de los niveles de insatisfacción del usuario externo del Instituto Nacional de Salud del Niño, Lima, Perú. Materiales y métodos. Estudio observacional basado en un programa para la evaluación de encuestas de usuarios en salud (SEEUS). La población estuvo conformada por todos los acompañantes del usuario externo en consulta ambulatoria, hospitalización y emergencia en los años 2008, 2009 y 2010. Para el cálculo del tamaño muestral se empleó la proporción de usuarios insatisfechos de cada servicio del año anterior, con un nivel de confianza del 95\% y un error estimado del 5\%. Resultados. Se obtuvo una muestra de 2051 acompañantes, en la cual se constató una tendencia decreciente de los niveles de insatisfacción en consulta ambulatoria, y un aumento de la insatisfacción en la dimensión capacidades médicas en hospitalización y emergencia. Se encontró además, índices de insatisfacción menores de 0,20 en las dimensiones capacidades médicas en consulta ambulatoria. En contraste, los mayores índices de insatisfacción se encontraron en las dimensiones de higiene durante los años $2009(0,25)$ y $2010(0,25)$, y otros en emergencia durante el $2009(0,25)$. Conclusiones. Las dimensiones en las que hubo un incremento en el índice de insatisfacción fueron aspectos tangibles, higiene y otros en hospitalización y emergencia. Por otra parte, la dimensión capacidades médicas en consulta ambulatoria mostró una disminución de los niveles de insatisfacción durante el periodo evaluado.

Palabras clave: Satisfacción del paciente; Calidad de la atención de salud; Salud del niño; Hospitales públicos; Perú. (fuente: DeCS BIREME).

\section{LEVELS OF DISSATISFACTION OF EXTERNAL USERS OF THE INSTITUTO NACIONAL DE SALUD DEL NIÑO (NATIONAL INSTITUTE OF CHILD HEALTH) IN LIMA, PERU}

\section{ABSTRACT}

Objectives. To determine the changes in the levels of dissatisfaction of the external users of the Instituto Nacional de Salud del Niño, in Lima, Peru. Materials and methods. Observational study based on a software to evaluate surveys completed by health care users (Software de Evaluación de Encuestas de Usuarios en Salud - SEEUS). The population consisted of every person accompanying external users to outpatient visits, inpatient visits or emergency treatment in the years 2008, 2009 and 2010. To calculate the sample size, last year's proportion of users dissatisfied with each service was used, with an estimated error of $5 \%$ at a $95 \%$ confidence level Results. A sample of 2051 people was obtained, which revealed a decreasing trend in the levels of dissatisfaction with outpatient visits, and an increased dissatisfaction in the medical capabilities dimension for hospitalization and emergency services. In addition, a dissatisfaction index lower than 0.20 was registered in the medical capabilities dimension for outpatient visits. In contrast, the highest dissatisfaction indexes were registered in the hygiene dimension for 2009 (0.25) and 2010 (0.25), and in the others dimension for emergency services in 2009 (0.25). Conclusions. The dimensions showing an increase in the dissatisfaction index were tangible aspects, hygiene and others for hospitalization and emergency services. On the other hand, the medical capabilities dimension for outpatient visits showed a decrease in the level of dissatisfaction over the evaluated period.

Key words: Patient satisfaction; Quality of health care; Health Child; Public Hospitals; Peru. (Source: MeSH NLM).

Instituto Nacional de Salud del Niño. Lima, Perú.

Facultad de Medicina, Universidad Nacional Mayor de San Marcos. Lima, Perú.

Facultad de Medicina "Alberto Hurtado", Universidad Peruana Cayetano Heredia. Lima, Perú.

Médico doctor en Medicina; ${ }^{\mathrm{b}}$ médico magíster en Medicina; ${ }^{\mathrm{c}}$ enfermera; ${ }^{\mathrm{d}}$ estadístico

Recibido: 06-06-12 Aprobado: 31-10-12

* Los resultados de este estudio fueron presentados parcialmente en la XLIX Reunión Anual de la Sociedad Latinoamericana de Investigación Pediátrica, 2011; Guanajuato, México.

Citar como: Shimabuku R, Huicho L, Fernández D, Nakachi G, Maldonado R, Barrientos A. Niveles de insatisfacción del usuario externo en el Instituto Nacional de Salud del Niño de Lima, Perú. Rev Peru Med Exp Salud Publica. 2012;29(4):483-9. 


\section{INTRODUCCIÓN}

Elevar los niveles de salud de una población implica asegurar la calidad de la atención, lo que a su vez está relacionado con la satisfacción del paciente. Se pone cada vez mayor énfasis a la calidad de los cuidados hospitalarios de los niños, en especial en países de recursos limitados ${ }^{(1-4)}$, pero también en países más desarrollados ${ }^{(5)}$. En concordancia con ello, la Organización Mundial de la Salud convocó a una reunión en Bali para revisar el tema y compartir la experiencia de dichos países ${ }^{(6)}$. Como resultado de dicha reunión y de las experiencias de los diversos países, se desarrolló un instrumento genérico de evaluación de la calidad de atención en establecimientos de salud de nivel referencial, el cual se basa en el marco clásico de calidad de atención que involucra estructura, procesos y resultados y que puede ser adaptado a las necesidades locales ${ }^{(6)}$.

Por lo menos desde 1994 los gobiernos de la región de las Américas han abordado el tema de calidad en los servicios básicos de salud como parte de los procesos de reforma del sector, reflejando su relevancia tanto para el sector público como para el privado ${ }^{(7,8)}$.

El marco clásico para la evaluación de la calidad y seguridad de la asistencia sanitaria incluye criterios que se pueden clasificar como pertenecientes a la estructura, a los procesos y a los resultados ${ }^{(9)}$, a lo que habría que agregar el criterio de accesibilidad ${ }^{(10)}$. Conocer el grado de satisfacción (o insatisfacción) del paciente (usuario externo) constituye un elemento necesario para garantizar la calidad, y debe ser uno de los indicadores reportados por los establecimientos de salud.

Calidad en los servicios de salud y satisfacción del paciente están estrechamente relacionados. El grado de satisfacción del acompañante del paciente pediátrico cumple una función importante en la atención médica. Se trata, en primer lugar, de un juicio sobre la calidad de la atención, que incorpora las expectativas y percepciones del paciente. En segundo lugar, se trata de un resultado de la atención: un aspecto del bienestar psicológico del paciente y también un signo de éxito para la institución sanitaria. En tercer lugar, la satisfacción del paciente asegura que cumpla su tratamiento, alentando a los pacientes en la búsqueda de atención y para que colaboren en lograr su mejoría (11). Por extensión, mala calidad de la atención se asocia con insatisfacción del paciente, lo cual se refleja en quejas, reclamos y denuncias en los medios de comunicación, impacto en la sociedad, desprestigio de las instituciones $y$, en algunos casos, en procesos judiciales ${ }^{(12-14)}$.

La evaluación de la insatisfacción debería contribuir al planteamiento de mejoras en la atención hospitalaria, además de servir de referente de atención para otros establecimientos de salud.

El objetivo del presente estudio fue evaluar los cambios en los resultados de las encuestas realizadas sobre los niveles de insatisfacción del usuario externo del Instituto Nacional de Salud del Niño (INSN), de Lima, Perú, entre los años 2008 al 2010. El INSN es un establecimiento de salud del más alto nivel de complejidad según la Norma Técnica de Categorías de establecimientos de salud del MINSA (III-2) dedicado exclusivamente a la atención de niños y adolescentes menores de 18 años.

\section{MATERIALES Y MÉTODOS}

Se presenta un estudio observacional, en el cual se empleó la encuesta para la evaluación del índice de insatisfacción del usuario denominada software para la evaluación de encuestas de usuarios en salud (SEEUS) (15); este programa ha sido validado por el Ministerio de Salud del Perú y es usado para actividades de supervisión de establecimientos de salud para evaluar el grado de satisfacción de los usuarios en los servicios de salud ${ }^{(16)}$.

\section{POBLACIÓN Y MUESTRA}

La población estuvo conformada por los usuarios externos (padres y acompañante del paciente) de las áreas de consulta ambulatoria, hospitalización y emergencia del INSN en los años 2008, 2009 y 2010. Para la toma de la muestra de estudio se consideró únicamente un padre o acompañante por cada menor en cada atención (en las áreas de consulta y emergencia) o por cada menor hospitalizado. Para el cálculo del tamaño de la muestra se fijó el nivel de confianza en 95\%, y el margen de error esperado en $5 \%$; la proporción empleada fue aquella que correspondía al año inmediatamente anterior en cada servicio $(0,16 ; 0,22$ y 0,15 en consulta externa; 0,$17 ; 0,19$ y 0,24 en emergencia; 0,$21 ; 0,21$ y 0,23 en hospitalización). Se procuró sistematizar la toma de muestra, para lo cual se entrevistó a uno de cada dos usuarios externos en el periodo julio-agosto de cada año.

\section{PROCEDIMIENTOS}

La entrevista a los usuarios externos mayores de 18 años que aceptaron ser entrevistados se efectuó a la salida de la consulta externa, previa verificación de haber recibido atención ambulatoria a través de la inspección de recetas y órdenes de exámenes auxiliares correspondientes al mismo día de la entrevista. Similar procedimiento se aplicó en emergencia. En hospitalización se entrevistó a la madre o tutor acompañante, la duración de cada encuesta fue de quince minutos aproximadamente. La recolección 
de datos fue realizada por el personal de orientación, en forma anónima y sin uniforme. Dicho personal recibió capacitación previa sobre técnicas de entrevista por parte de miembros de la Oficina de Gestión de la Calidad del INSN.

\section{INSTRUMENTOS}

El SEEUS evalúa la insatisfacción en seis dimensiones (capacidades médicas, higiene, aspectos tangibles, trato al usuario, tiempo, y otros) ${ }^{(15)}$, para el análisis se evaluaron las seis dimensiones en cada una de las áreas de interés durante los tres años que duró el estudio. En aspectos tangibles se analizó: equipos, apariencia, señalización y confort. En capacidades médicas se indagó sobre explicación del diagnóstico, explicación del tratamiento, explicación de exámenes, capacidad de comunicación, respuestas a interrogantes, conocimiento de la enfermedad y dedicación al paciente. En trato del personal se preguntó sobre trato en economía (caja), trato en admisión y trato del médico. En la dimensión tiempo se averiguó sobre tiempo de atención, tiempo en caja, tiempo de atención por enfermeras, tiempo en trámite de atención, tiempo en farmacia, tiempo de laboratorio y tiempo de servicios al diagnóstico. En higiene se interrogó sobre la limpieza del local. La dimensión otros comprende: privilegios, disponibilidad de medicinas, disponibilidad de laboratorios, disponibilidad de servicios al diagnóstico y privacidad de atención. El puntaje mínimo que se puede obtener en el SEEUS es 0 y el máximo es $1^{(15)}$, con lo cual se clasifican el índice de insatisfacción de cada dimensión en leve $(0-0,20)$, moderado $(>0,20-0,40)$ y, severo $(>0,40-1)$. El último indicador en las tres áreas evaluadas fue "si la causa por la que se vino a atender fue solucionada".

\section{ANÁLISIS ESTADÍSTICO}

Se realizaron análisis descriptivos que incluyeron medidas de frecuencia, porcentajes y promedios. Para asociar los niveles de insatisfacción, medido por el indicador "solución del motivo de atención", se aplicó la prueba de chi cuadrado con corrección de Yates o prueba exacta de Fisher, según corresponda. Para el procesamiento de los datos se usó el paquete estadístico SPSS v.15.0. Se consideró un valor $p<0,05$ como significativo.

\section{ASPECTOS ÉTICOS}

Las encuestas se aplicaron a los padres o tutores que expresaron su deseo de participar voluntariamente (consentimiento verbal). Se garantizó la confidencialidad de los datos obtenidos y se preservó la identidad de los participantes. El Proyecto fue aprobado por el Comité de Ética Institucional del Instituto de Salud del Niño (código IRB0003278 Instituto de Salud del Niño IRB \#1).

\section{RESULTADOS}

El tamaño final de la muestra fue de 2051 encuestados, distribuidos de la siguiente manera: 725 encuestados en consulta externa (203 en el 2008, 260 en el 2009, 262 en el 2010); 680 encuestados en el servicio de emergencia (207 en el 2008, 230 en el 2009, 243 en el 2010) y 646 encuestados en los servicios de hospitalización (175 en el 2008, 205 en el 2009, 266 en el 2010). El 98,4\% de los acompañantes fueron de sexo femenino, está proporción fue más notoria en emergencia $(93,8 \%)$. Se encontró una mayor proporción de acompañantes con grado de instrucción secundaria (62,7\%); en tanto que la procedencia encontrada fue mayoritariamente urbana $(90,8 \%)$. Cabe señalar que el $59,6 \%$ de los encuestados manifestó que acudía por primera vez al INSN, valor que se hace más relevante en el área de consulta ambulatoria donde el $78,3 \%$ de los encuestados manifestó que aquella era su primera atención en este

Tabla 1. Características de los acompañantes del usuario externo del INSN, Lima, Perú. 2008-2010.

\begin{tabular}{|c|c|c|c|c|c|c|c|}
\hline \multicolumn{2}{|c|}{ Caracteristicas del acompañante } & \multicolumn{2}{|c|}{$\begin{array}{c}\text { Consulta Externa } \\
\left(\mathrm{N} .{ }^{\circ}=725\right)\end{array}$} & \multicolumn{2}{|c|}{$\begin{array}{l}\text { Hospitalización } \\
\left(\mathrm{N} .{ }^{\circ}=646\right)\end{array}$} & \multicolumn{2}{|c|}{$\begin{array}{c}\text { Emergencia } \\
\left(\mathrm{N}^{\circ}{ }^{\circ}=680\right)\end{array}$} \\
\hline & & N. ${ }^{\circ}$ & $(\%)$ & N. ${ }^{\circ}$ & $(\%)$ & N. ${ }^{\circ}$ & $(\%)$ \\
\hline \multirow[b]{2}{*}{ Sexo } & Femenino & 674 & $(93,4)$ & 522 & $(81,6)$ & 638 & $(93,8)$ \\
\hline & Masculino & 48 & $(6,6)$ & 118 & $(18,4)$ & 42 & $(6,2)$ \\
\hline \multirow{4}{*}{ Grado de Instrucción } & Ninguno & 2 & $(0,3)$ & 5 & $(0,8)$ & 2 & $(0,3)$ \\
\hline & Primaria & 95 & $(13,3)$ & 87 & $(13,5)$ & 47 & $(6,9)$ \\
\hline & Secundaria & 438 & $(61,2)$ & 430 & $(66,8)$ & 419 & $(61,7)$ \\
\hline & Superior & 181 & $(25,3)$ & 122 & $(18,9)$ & 211 & $(31,1)$ \\
\hline \multirow{5}{*}{ Tiempo de Espera } & Muy largo & 94 & $(13,1)$ & 86 & $(13,4)$ & 11 & $(1,6)$ \\
\hline & Largo & 377 & $(52,5)$ & 247 & $(38,4)$ & 132 & $(19,4)$ \\
\hline & Adecuado & 212 & $(29,5)$ & 244 & $(37,9)$ & 379 & $(55,8)$ \\
\hline & Corto & 34 & $(4,7)$ & 62 & $(9,6)$ & 153 & $(22,5)$ \\
\hline & Muy corto & 1 & $(0,1)$ & 4 & $(0,6)$ & 4 & $(0,6)$ \\
\hline \multirow{2}{*}{ Procedencia } & Lima Provincia & 659 & $(91,1)$ & 551 & $(85,8)$ & 652 & $(95,9)$ \\
\hline & Otras regiones & 64 & $(8,9)$ & 91 & $(14,2)$ & 28 & $(4,1)$ \\
\hline \multirow{2}{*}{ Atención previa en el INSN } & $\mathrm{Si}$ & 157 & $(21,7)$ & 389 & $(60,3)$ & 279 & $(0.41)$ \\
\hline & No & 566 & $(78,3)$ & 256 & $(39,7)$ & 401 & $(0.59)$ \\
\hline Edad en años & Media $\pm \mathrm{DE}$ & \multicolumn{2}{|c|}{$33,3 \pm 9,2$} & \multicolumn{2}{|c|}{$34,2 \pm 9,4$} & \multicolumn{2}{|c|}{$32,4 \pm 8,2$} \\
\hline
\end{tabular}

Nota: El tiempo de espera descrito está referido al tiempo que transcurre desde que el usuario llega a la institución hasta que es atendido. INSN: Instituto Nacional de Salud del Niño. 
establecimiento (Tabla 1). En nuestra muestra, el $80 \%$ de los menores hospitalizados (incluyendo los sometidos a intervenciones quirúrgicas) contaban con Seguro Integral de Salud (SIS), el cual cubrió su atención; en contraste, solo el $15 \%$ de los pacientes ambulatorios y el $10 \%$ de los que acudieron a Emergencia tenían SIS.

En la Tabla 2 se muestra los niveles de insatisfacción en las seis dimensiones, de acuerdo a cada área estudiada. Se encontró que los índices de insatisfacción globales correspondientes a los años 2008, 2009 y 2010 en consulta ambulatoria fueron de 0,22, 0,15 y 0,09 respectivamente; en hospitalización fueron 0,21, 0,23 y 0,22 ; y en emergencia fueron $0,19,0,24$ y 0,24, lo cual evidencia una marcada disminución de la insatisfacción en el área de consulta ambulatoria, en tanto que el índice de insatisfacción se mantuvo en hospitalización y mostró un ligero aumento en el área de emergencia.

Siguiendo con este análisis, ahora en cada una de las dimensiones según servicio, se encontró que en el área de consulta ambulatoria el índice de insatisfacción de usuarios disminuyó en las dimensiones aspectos tangibles, capacidades médicas, trato del personal e higiene; y solo se evidenció incremento en la dimensión otros. En tanto que, en el área de hospitalización se pudo ver que el índice de insatisfacción para la dimensión trato al personal disminuyó para cada año de estudio; por el contrario, se pudo ver un incremento en la dimensión capacidades médicas. En el área de emergencia, se encontraron igualmente incrementos de este índice en las dimensiones capacidades médicas e higiene. Todas las demás dimensiones se mantuvieron en el tiempo.
En el análisis anual de cada una de las dimensiones, en las tres áreas, se verificó un nivel de insatisfacción moderado; en donde, de 54 campos evaluados el 83,3\% presentó un índice de insatisfacción moderada y el 16,7\% presentó un índice de insatisfacción leve, en ningún caso hubo nivel de insatisfacción severo. Se encontró que el menor nivel de insatisfacción de usuario se registró en el año 2010 en el área de consulta ambulatoria en la dimensión higiene $(0,01 ; \mathrm{IC}: 0,00-0,02)$; en contraste, esta misma dimensión registró el mayor índice de insatisfacción $(0,25$; IC:0,20-0,30) en área de emergencia en el mismo año. Cabe señalar además, que tanto en el área de hospitalización como en el de emergencia, el menor nivel de insatisfacción fue constatado en la dimensión capacidades médicas en el año 2008; sin embargo, esta dimensión decae para el año 2010, como se señala líneas arriba. En resumen, de los nueve indicadores que tuvieron los menores índices de insatisfacción en años consecutivos, corresponden a la dimensión capacidades médicas (cinco de nueve), trato del personal, (dos de nueve) tiempo (uno de nueve), e higiene (uno de nueve).

En cuanto al grado de instrucción, se encontró que solo existió asociación en el servicio de emergencia durante el año $2010(p=0,014)$, donde se evidencia mayores porcentajes de insatisfacción en secundaria y superior y menores en primaria $(57,6 \%, 30,3 \%$ y $12,1 \%)$. Cuando comparamos la variable "si la causa por la que se vino a atender fue solucionado", encontramos que en el área de consulta ambulatoria durante los años 2008, 2009 y 2010 , los resultados fueron significativos $(p<0,05)$ para los indicadores "tiempo de la consulta", "el médico le explicó lo que tuvo", "respondió a las preguntas sobre su

Tabla 2. Dimensiones e índices de insatisfacción del usuario externo.

\begin{tabular}{cccccccccccc}
\hline \multirow{2}{*}{ Dimensiones Consulta ambulatoria } & \multicolumn{3}{c}{ Hospitalización } & \multicolumn{3}{c}{ Emergencia } \\
\cline { 2 - 10 } & $\mathbf{2 0 0 8}$ & $\mathbf{2 0 0 9}$ & $\mathbf{2 0 1 0}$ & $\mathbf{2 0 0 8}$ & $\mathbf{2 0 0 9}$ & $\mathbf{2 0 1 0}$ & $\mathbf{2 0 0 8}$ & $\mathbf{2 0 0 9}$ & $\mathbf{2 0 1 0}$ \\
\hline \multirow{2}{*}{$\begin{array}{c}\text { Aspectos } \\
\text { tangibles }\end{array}$} & 0,24 & 0,22 & 0,20 & 0,23 & 0,25 & 0,25 & 0,23 & 0,25 & 0,25 \\
Capacidades & $(0,18-0,29)$ & $(0,17-0,27)$ & $(0,15-0,25)$ & $(0,17-0,30)$ & $(0,19-0,31)$ & $(0,19-0,29)$ & $(0,18-0,29)$ & $(0,19-0,30)$ & $(0,19-0,30)$ \\
médicas & 0,12 & 0,09 & 0,05 & 0,10 & 0,20 & 0,22 & 0,15 & 0,21 & 0,23 \\
Trato del & $0,08-0,17)$ & $(0,06-0,13)$ & $(0,02-0,08)$ & $(0,06-0,15)$ & $(0,15-0,26)$ & $(0,17-0,27)$ & $(0,09-0,19)$ & $(0,16-0,26)$ & $(0,17-0,28)$ \\
personal & 0,23 & 0,21 & 0,21 & 0,24 & 0,21 & 0,19 & 0,22 & 0,22 & 0,24 \\
Tiempo & $0,17-0,29)$ & $(0,16-0,26)$ & $(0,16-0,26)$ & $(0,17-0,29)$ & $(0,15-0,26)$ & $(0,14-0,23)$ & $(0,16-0,28)$ & $(0,17-0,28)$ & $(0,19-0,29)$ \\
& $(0,13-0,24)$ & $(0,14-0,23)$ & $(0,13-0,23)$ & $(0,16-0,28)$ & $(0,16-0,27)$ & $(0,18-0,29)$ & $(0,15-0,26)$ & $(0,17-0,28)$ & $(0,15-0,25)$ \\
Higiene & 0,24 & 0,25 & 0,01 & 0,25 & 0,24 & 0,25 & 0,29 & 0,25 & 0,25 \\
& $(0,18-0,30)$ & $0,19-0,30)$ & $(0,00-0,02)$ & $(0,18-0,31)$ & $(0,18-0,30)$ & $(0,19-0,30)$ & $(0,18-0,30)$ & $(0,19-0,30)$ & $(0,20-0,30)$ \\
Otros & 0,19 & 0,19 & 0,21 & 0,20 & 0,23 & 0,23 & 0,22 & 0,25 & 0,24 \\
& $(0,14-0,25)$ & $(0,14-0,24)$ & $(0,16-0,26)$ & $(0,14-0,263)$ & $(0,17-0,28)$ & $(0,18-0,28)$ & $(0,16-0,28)$ & $(0,19-0,30)$ & $(0,19-0,30)$ \\
\hline
\end{tabular}

Nota: se muestran los índices de insatisfacción e intervalos de confianza al 95\%. 
enfermedad", y "conocía como tratar su enfermedad"; en donde, el primer indicador corresponde a la dimensión tiempo y los restantes a la dimensión capacidades médicas. En hospitalización, fueron significativos $(p<0,05)$, durante los años 2009 y 2010, los indicadores "trato del técnico de enfermería", "trato del personal de laboratorio" y "frecuencia de la sala de hospitalización"; donde los dos primeros indicadores correspondían a la dimensión trato del personal, y el último a la dimensión higiene. En Emergencia, fueron significativos $(p<0,05)$ los indicadores "el médico le explicó los exámenes" los años 2009 y 2010, y "conocía como tratar su enfermedad" los años 2008, 2009 y 2010. Los dos indicadores corresponden a la dimensión capacidades médicas.

\section{DISCUSIÓN}

La evaluación de la calidad tiene como propósito entender la magnitud y distribución de la calidad y cómo se ve afectada por las características de la sociedad, de la institución, del personal de salud y por las categorías de pacientes ${ }^{(17)}$. Desde el año 2008 se inició en el INSN la evaluación y supervisión de la calidad con el indicador satisfacción del usuario externo. Su finalidad es obtener información continua de la práctica de la atención sanitaria, con el objetivo de determinar si está logrando o no sus objetivos, de acuerdo a los planes ejecutados, y determinar las causas de las deficiencias para realizar las correcciones oportunamente, y verificar luego las mejoras logradas.

La mayoría de pacientes incluidos en el presente estudio procedían de Lima, lo que se verificó en las tres áreas de atención (consulta ambulatoria, hospitalización y emergencia). Esto refleja la composición fundamentalmente urbana del país, pero también la dificultad de acceder a los servicios de salud de las zonas alejadas de la capital.

La mayoría de los pacientes hospitalizados tenía cobertura del Sistema de Seguro Integral, incluyendo los sometidos a intervenciones quirúrgicas. Esta es una variable que hay que tener en consideración al interpretar los resultados, pues aquellos que pagan por un servicio de salud podrían tener expectativas y percepciones diferentes en relación a los que tienen cobertura de un seguro público. Por lo tanto, la generalización de nuestros resultados a toda la población que se atiende en el Instituto debe ser considerada con cautela.

En consulta ambulatoria, los aspectos de la dimensión capacidades médicas mostraron el menor nivel de insatisfacción. Una posible explicación para este resultado es que se trata de pacientes cuyas causas de consulta son frecuentemente más sencillas y no requieren la urgencia de atención en emergencia o la demanda continua y muchas veces prolongada de equipamiento y personal que sí se requieren en hospitalización. Sus expectativas y necesidades son menores, lo que corrobora los resultados del estudio de De Oliveira, en el cual se encontró un buen nivel de satisfacción en pacientes ambulatorios (18). Las capacidades médicas o dimensión técnica en la que el usuario aprueba los conocimientos del personal de salud para resolver el motivo por el que acude al hospital es la que alcanza los menores niveles de insatisfacción, lo que también está en concordancia con lo descrito por Gogeascoechea et al. ${ }^{(19)}$. Al igual que en otros informes en hospitales en el Perú, la consulta ambulatoria mostró menores índices de insatisfacción que la encontrada en las áreas de hospitalización y emergencia ${ }^{(20)}$. De manera similar, en un estudio en México por Puente Rosas et al. se hallaron resultados semejantes, donde los porcentajes de aprobación tuvieron una tendencia mayor en atención ambulatoria con respecto a la atención hospitalaria ${ }^{(21)}$.

Existe la idea generalizada de que en los hospitales públicos la atención es de menor calidad que la de los establecimientos privados, pero en estos últimos la insatisfacción puede estar más asociada a sus costos de atención que a factores como los evaluados por nosotros. En el estudio de Ramírez-Sánchez et al. se puso de manifiesto que cuando la atención en los servicios privados fue definida por los usuarios como mala, ello se debió básicamente a los altos costos de esta ${ }^{(22)}$. Hay un estudio singular realizado en Pakistán en que los pacientes percibieron que la calidad en los hospitales públicos era superior a la de los hospitales privados ${ }^{(23)}$. En el estudio de De Oliveira, los pacientes con bajos niveles de ingresos y educación, y que no encuentran otras opciones de atención, mostraron altos niveles de satisfacción ${ }^{(18)}$.

El nivel de insatisfacción en el servicio de emergencia puede ser explicada en buena parte por el hecho de que la atención se debe con más frecuencia a enfermedades o accidentes que ponen en riesgo inminente la vida del paciente, atención que requiere prontitud, recursos especiales y resultados inmediatos en la percepción de los usuarios. En esta área hay que tener en cuenta el estado emocional de los acompañantes de los pacientes, que valoran mucho el tiempo de espera el cual quisieran que se traduzca en rapidez de la atención, como lo demuestra el estudio de Wong et al. en Singapore ${ }^{(12)}$.

Usualmente los usuarios de nivel superior muestran mayores niveles de insatisfacción ${ }^{(24,25)}$, algo que 
nosotros no encontramos en ninguna área estudiada, en contraste con resultados de estudios realizados en Trujillo y Arequipa, en los que sí se encontraron que los niveles de insatisfacción fueron mayores en encuestados con educación superior, lo que refleja mayores expectativas y conocimiento de sus derechos por parte de los usuarios. En general, el grado de instrucción es un indicador del nivel socioeconómico. Los de menor grado de instrucción se relacionan con los quintiles de riqueza más bajos ${ }^{(26)}$.

Los pacientes hospitalizados, debido a la gravedad del paciente o a la incertidumbre sobre su diagnóstico y pronóstico, están sometidos con frecuencia a situaciones de estrés y de frustración diaria, lo que no ocurre con la misma intensidad ni frecuencia en consulta ambulatoria. En esta área, los encuestados valoran la información diaria y desean permanecer el mayor tiempo posible con el niño y tener un ambiente más amigable ${ }^{(27)}$. En nuestro estudio la mayoría de niños atendidos fueron menores de 5 años (51\%) y comprensiblemente, las personas responsables de su cuidado y las que fueron encuestadas fueron mujeres entre 30 a 34 años.

La principal ventaja de este estudio es que compara los niveles de insatisfacción para tres periodos anuales consecutivos, y brinda información útil sobre la evolución de las dimensiones con mayores fortalezas y aquellas que requieren mayor trabajo. Esta información constituyó un instrumento importante que la gestión del INSN utilizó para planificar estrategias de mejora y fortalecimiento de los servicios en las diversas áreas asistenciales mencionadas. Entre las medidas adoptadas para mejorar la calidad del servicio se incluyen medidas de mejoramiento de la infraestructura, de la señalización, mejoras en diversos pasos del proceso de atención para reducir el tiempo de espera, la reubicación del área de admisión y del ambiente de seguro integral de salud para reducir el hacinamiento, el acceso a la farmacia, el dictado de cursos de buen trato al usuario y de clima organizacional, entre muchas otras.
El estudio tiene limitaciones, la primera es la posible presencia de un sesgo de medición ya que en el recojo de muestra participaron entrevistadores institucionales (personal de orientación); sin embargo, cabe señalar que dicho personal es el que tiene un menor vinculo institucional lo cual minimiza la posibilidad de parcializarse a favor de la institución. Otra limitación fue que en nuestro estudio no se incluyó el uso de otros instrumentos de verificación de la calidad en las diversas áreas, instrumentos comúnmente utilizados en la evaluación del tiempo real de espera, del manejo de casos, verificación de la infraestructura, de la disponibilidad de equipos, medicamentos e insumos, entre otros; ello no permite controlar la subjetividad del usuario externo producida en función a si el motivo por el cual acudió en busca de atención fue solucionado o no. En estudios futuros se puede combinar el instrumento que utilizamos con estos mencionados, de modo que podamos obtener una evaluación más integral de la calidad del servicio brindado.

En conclusión, las dimensiones que tuvieron un mayor índice de insatisfacción fueron aspectos tangibles, higiene $y$ otros en hospitalización y emergencia durante el periodo de estudio frente a los cuales se han efectuado mejoras cuyas repercusiones se están evaluando. La dimensión capacidades médicas en consulta ambulatoria mostró los menores niveles de insatisfacción a lo largo del tiempo, constituyendo una fortaleza que debe utilizarse para mejorar aún más el grado de satisfacción del usuario externo.

Contribuciones de autoria: RS y DF contribuyeron en la organización del estudio; DF y RM en la recolección de datos, además, todos los autores participaron en la elaboración del proyecto, análisis de datos, elaboración de borradores del manuscrito y revisión y aprobación de la versión final

Fuentes de financiamiento: autofinanciado.

Conflictos de interés: los autores declaran que entre ellos se encuentran directivos del INSN, que es la institución evaluada.

\section{REFERENCIAS BIBLIOGRÁFICAS}

1. Ntoburi S, Hutchings A, Sanderson C, Carpenter J, Weber M, English M. Development of paediatric quality inpatient indicators for low-income countries - A Delphi study. BMC Paediatr. 2010;10:90.

2. Duke T, Tamburlini G. Improving the quality of paediatric care in peripheral hospitals in developing countries. Arch Dis Child. 2003; 88: 563-565.
3. Nolan T, Angos P, Cunha AJLA, Muhe L, Qazi S, Simoes EAF Tamburlini G Weber M, Pierce NF. Quality of hospital care for seriously ill children in lessdeveloped countries. Lancet. 2001; 357 (9250): 106-110.

4. Andaleeb S. Caring for children; a model of healthcare service quality in Bangladesh. Int J Qual Health Care. 2008; 30(5): 339-345.
5. Duke T, Keshishiyan E, Kuttumuratova A, Ostergren M, Ryumina I, Stassi E, Weber MW, Tamburlini G. Quality of hospital care for children in Kazakhstan, Republic of Moldova, and Russia: systematic observational assessment. Lancet. 2006; 367 (9514): 919-925.

6. Campbell H, Duke T, Weber M, English M, Carai S, Tamburlini G. Global Initiatives for Improving Hospital 
Care for Children: State of the Art and Future Prospects. Pediatrics. 2008; 121(4):e984-992.

7. Organización Panamericana de la Salud. Programa de garantía y mejoramiento de calidad de los servicios de salud en América Latina y el Caribe. Washington, DC: OPS; 1999.

8. Ross AG, Zeballos JL, Infante A. La calidad y la reforma del sector de la salud en América Latina y el Caribe. Rev Panam Salud Publica. 2000 ; 8(12): 93-98.

9. Donabedian A. Evaluating the Quality of Medical Care. Milbank Q. 1966; 44(3): 166-203.

10. Zurita Garza B. Calidad de la atención en salud. An Fac Med Lima. 1996; 57 (4): 1-9.

11. Donabedian A. The Lichfield lecture. Quality assurance on health care: consume's role. Qual Health Care 1992; $1: 247-51$.

12. Wong LL, Ooi SBS, Gogh LG. Patient'complaints in a hospital emergency department in Singapore. Singapore Med J. 2007; 48(11):990.

13. Taylor DMcD, Wolfe RS, Cameron PA. Analysis of complaints by patients attending Victorian hospitals. Med J Aust. 2004; 181(1):31-35.

14. Giugliani C, Gault N, Fares V, Jegu J, Eleni dit Trolli S, Biga J, Vidal-Trecan G. Evolution of patients' complaints in a French university hospital: is there a contribution of a law regarding patients' rights ?. BMC Health Serv Res. 2009; 9:141.
15. PHR plus - USAID. Manual del usuario del SEEUS. Perú, 2005.

16. Propuesta metodológica de medición de un índice de insatisfacción como indicador referencial de la calidad de los servicios de salud. Ministerio de Salud del Perú, 2002.

17. Donabedian A. The Assessment of Technology and Quality: A Comparative Study of Certainties and Ambiguities. Intl J of Technology Assessment in Health Care. 1988; 4:487-96.

18. De Oliveira DF, Arieta CEL, Temporini ER, Kara-José N. Quality of health care: patient satisfaction in a university hospital. Arq Bras Oftalmol. 2006; 69 (5): 731-6.

19. Gogeascoechea-Trejo MC, PavónLeón P, Blásquez-Morales MSL, Blásquez-Domínguez CR, RabatteSuárez I. Satisfacción y trato al usuario en un hospital universitario. Rev Med UV. 2009; 9(1):6-10.

20. Ministerio de Salud del Perú. Índice de insatisfacción de los usuarios de los servicios de salud. Documento de discusión, Ministerio de Salud, Proyecto 2000.

21. Puentes Rosas E, Gómez Dantés O, Garrido La Torre F. Trato a los usuarios en los servicios públicos de salud en México. Rev Panam Salud Publica/Pan Am J Public Health. 2006; 19(6). 394402.

22. Ramírez-Sanchez TJ, Nájera-Aguilar P, Nigenda-López G. Percepción de la calidad de la atención de los servicios de salud en México: perspectivas de los usuarios. Salud Publica Mex. 1998; 40 (1):3-12.

23. Shabbir S, Kaufmann HR, Shehzad M. Service quality, word of mouth and trust: Drivers to achieve patient satisfaction. Sci Res Essays. 2010; 5 (17): 2457-2462.

24. Seclén-Palacin J, Darras C. Satisfacción de usuarios de los servicios de salud: Factores sociodemográficos y de accesibilidad asociados. Perú, 2000. An Fac Med Lima. 2005; 66(2):127-140.

25. Ortiz Espinosa RM, Muñoz Juárez $S$, Lechuga Martin del Campo D, Torres Carreño E. Consulta externa en instituciones de salud de Hidalgo, México, según la opinión de los usuarios. Rev Panam Salud Publica. 2003; 13(4): 229-238.

26. Perú, Presidencia del Consejo de Ministros, Instituto Nacional de Estadística e Informática (INEI). Perú, Encuesta Demográfica y de Salud Familiar-Endes Continua, 2010, Informe Principal. Lima: INEI; 2011.

27. Goldbloom RB. The evolution of paediatric hospitals. Arch Dis Child. 2005; 90:113-4.

Correspondencia: Roberto Shimabuku Dirección: Av. Brasil 600, Breña. Lima 5, Perú.

Teléfono: (511) 3300066

Correoelectrónico: rshimabuku@isn.gob.pe

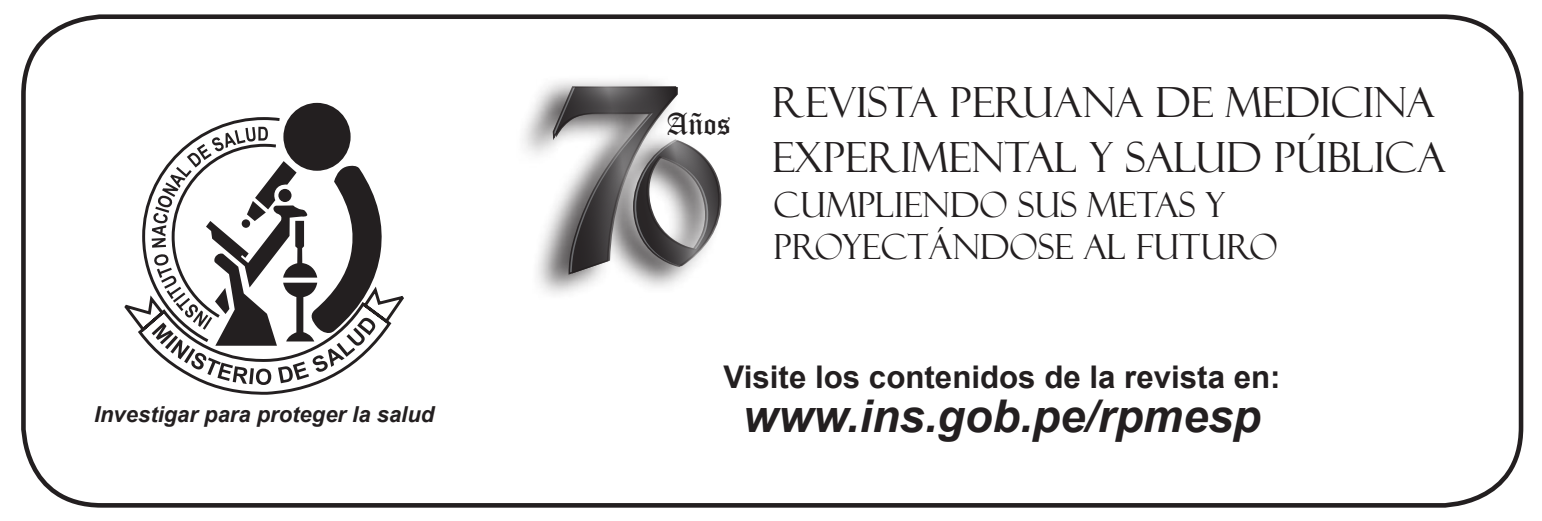

